\title{
EFFICACY OF 7 DAY COURSE OF AMBULATORY ORAL AMOXICILLIN FOR TREATING SEVERE PNEUMONIA IN 3-59 MONTHS OLD INDIAN CHILDREN- MULTICENTRIC RANDOMISED CONTROL TRIAL (ISPOT GROUP)
}

\author{
A. Patel ${ }^{1,2}$, L. Dhande ${ }^{1}$, A. Bang ${ }^{3}$, A. Malik ${ }^{4}$, L.R. Chelliah ${ }^{5}$, M. Singh ${ }^{6}$, S. Khadse ${ }^{7}$, ISPOT(IndiaCLEN \\ Severe Pneumonia Oral Therapy) \\ ${ }^{1}$ Pediatrics, Indira Gandhi Government Medical College, ${ }^{2}$ Lata Medical Research Foundation, Nagpur, \\ ${ }^{3}$ MGIMS, Sewagram, ${ }^{4}$ J.N.Medical College, Aligarh Muslim University, Aligarh, ${ }^{5}$ Pediatrics Institute of \\ Child Health \& Hospital for Children, Madras Medical College, Chennai, ${ }^{6}$ Post Graduate Institute Medical \\ Education \& Research, Chandigarh, ${ }^{7}$ B.J. Medical College, Pune, India
}

Background and aims: Injectable penicillin and hospitalization is the recommended treatment for WHOdefined severe pneumonia in children aged 3-59 months. We determined if treatment with oral amoxicillin when administered for first 48 hours in the hospital followed by 5 days at home (hospital group) was equivalent to a 7 day ambulatory treatment (home group).

Methods: This multicentre, randomised open-label equivalency study was conducted at 6 centres in India. Children aged 3-59 months with severe pneumonia were screened $(\mathrm{n}=6451)$ for inclusion and randomised to home $(n=505)$ or hospital group $(n=499)$. Follow-up assessments were done at day 3, 5, 7 and 14 . Primary outcome was treatment failure (persistence of lower chest in drawing or fever or clinical deterioration) on day 7. Analyses were by per protocol and intention-to-treat.

Results: The per protocol analysis excluded 8 patients of home group, 4 from hospital for loss to follow up and 29 patients that left the hospital against medical advice. Both groups had similar baseline characteristics. Treatment failure was $8.5 \%$ vs $10.5 \%$ in home and hospital group respectively (risk difference $-1.5 \%, 95 \%$ CI -5.1 to $2.1 ; \mathrm{p}=0.4$ ) on day 7 , both with a single death. There was no difference in adjusted odds ratio of cumulative failure at $\leq 7$ days between the two groups

Conclusion: Home based treatment with oral amoxicillin was equivalent to hospital treatment for first 48 hours in selected children of WHO defined severe pneumonia. It can reduce referral, hospital admissions, its problems and costs so warrents inclusion in WHO recommendations. 